\title{
Molecular Analysis and Expression Patterns of the 14-3-3 Gene Family from Oryza Sativa
}

\author{
Yuan Yao, Ying Du, Lin Jiang and Jin-Yuan Liu* \\ Laboratory of Molecular Biology and MOE Laboratory of Protein Science, Department of Biological Sciences and Biotechnology, \\ Tsinghua University, Beijing, P. R. China
}

Received 21 July 2006, Accepted 15 December 2006

The ubiquitous family of 14-3-3 proteins functions as regulators in a variety of physiological processes. Eight rice 14-3-3 genes, designated OsGF14a through $h$, were identified from an exhaustive search of the genome database. Comparisons of deduced amino acid sequences reveal a high degree of identity among members of the OsGF14 family and reported Arabidopsis 14-3-3 proteins. A phylogenetic study indicates that OsGF14s contain both $\varepsilon$ and non- $\varepsilon$ forms, which is also confirmed by a structural analysis of $\mathrm{OsGF14}$ genes. Furthermore, transcripts of OsGF14b, OsGF14c, OsGF14d, OsGF14e, OsGF14f and OsGF14g were detected in rice tissues. Their different expression patterns, the different effects of environmental stresses and plant hormones on their transcription levels, and the different complementary phenotypes in yeast 14-33 mutants not only indicates that $O S G F 14 s$ are responsive to various stress conditions and regulated by multiple signaling pathways, but also suggests that functional similarity and diversity coexist among the members of OsGF14 family.

Keywords: Gene family, Oryza sativa, Semiquantitative RTPCR, Yeast function complementation, 14-3-3

\section{Introduction}

The 14-3-3 proteins are small $(\sim 30 \mathrm{kD})$, acidic proteins that form both homo- and heterodimers. Discovered 36 years ago,

\footnotetext{
Abbreviations: ET: ethephon, ABA: abscisic acid, SA: salicylic acid, IAA: indole-3-acetic acid, JA: jasmonic acid, 5-FOA: 5-fluoroorotic acid
}

*To whom correspondence should be addressed. Tel: +86-10-62772243; Fax: +86-10-62772243

E-mail: liujy@mail.tsinghua.edu.cn
14-3-3 proteins were originally characterized as major soluble proteins in the brain tissue of mammals and named on the basis of their electrophoretic mobilities (Fu et al., 2000). Now it has become clear that 14-3-3 proteins constitute a ubiquitous protein family present in virtually all eukaryotic organisms (Rosenquist et al., 2000). For instance, two, seven and fifteen 14-3-3 homologues were found in yeast, human and Arabidopsis, respectively (van Heusden et al., 1995; Rosenquist et al., 2001; Qi et al., 2005). 14-3-3 proteins from plants and animals have significantly similarity in amino acid sequence (commonly $50 \%$ identity or so), except for their N-terminal dimerization domains and the hyper-variable $\mathrm{C}$ termini (Wang and Shakes, 1996). The high level of conservation endows 143-3 proteins from different species with some general features, which is supported by the successful function complementation of four Arabidopsis homologues in yeast mutants devoid of endogenous 14-3-3 proteins (van Heusden et al., 1996). Currently, individual members of Arabidopsis and human 143-3 proteins are designated by Greek letters. 14-3-3 isoforms from some plant species are also named GF14, because the first reported plant 14-3-3 isoform, Arabidopsis GF14 $\omega$, was identified as a component of the protein/G box complex and thus designated "G-box Factor 14-3-3" (Lu et al., 1992). This designation scheme has been maintained in Arabidopsis, rice and maize.

In plants, 14-3-3 proteins are known to be involved in a large number of biological processes via interactions with numerous target proteins in a phosphorylation-dependent manner. The effects caused by 14-3-3 binding can vary from inactivation to activation of the enzymatic activity of the client, the degradation or protection from degradation of the client, and the movement of the client from one cellular location to another (Bachman et al., 1996; Muslin and Xing, 2000; Dougherty and Morrison, 2004). Several of the best characterized 14-3-3 clients include nitrate reductase (Bachman et al., 1996), sucrose-phosphate synthase (Toroser et al., 1998), plasma membrane $\mathrm{H}^{+}$-ATPase (Jahn et al., 1997), the transcription factor EmBP1 and VP1 (Schultz et al., 1998), 
the RSG transcription activators (Igarashi et al., 2001), a lipoxygenase from barley (Holtman et al., 2000), a membranebound ascorbate peroxidase (Zhang et al., 1997), and a tomato outward-rectifying $\mathrm{K}^{+}$channel (Booij et al., 1999).

Although recent work has uncovered many details of 14-33 proteins in plants, little attention to date has been focused on rice GF14s. The first studied rice 14-3-3 gene, SR14-3-3, was found to be induced in rice seedlings under salinity and cold stresses (Kidou et al., 1993). Another four isoforms available in the NCBI database were also identified as 14-3-3 proteins based on their amino acid sequence homology with known 14-3-3 proteins, and were designated as OsGF14b, c, d, and e. Fortunately, completion of the rice genome sequence enables a final determination of the gene numbers within a specific gene family and characterization of the expressed members. In the present study, we have raised the total number of OsGF14s identified to eight by data mining of the whole rice genome and homologous alignment. At least six members could be transcribed in the materials we examined. RT-PCR analysis showed that different patterns were exhibited by the expressed members of this family in terms of tissue distribution and response to stresses and phytohormones. Furthermore, six expressed OsGF14s exhibited different functional complementation abilities in yeast 14-3-3 mutants. These results suggest that the highly conserved OsGF14 proteins might play diverse roles in the developmental process and under stress conditions, in addition to their overlapping functions.

\section{Materials and Methods}

Chemical agents. The RNeasy Plant Mini Kit was purchased from QIAGEN GmbH (Hilden). The RT-PCR system was provided by Promega (Madison) and DNase I was purchased by Sigma (St. Louis). Taq polymerase and pMD18-T vector were bought from TaKaRa (Dalian). SD medium and all supplemental materials used in yeast culture were purchased from BD Biosciences (Palo Alto).

Plant materials. Rice (Oryza sativa L. cv. Zhonghua 10) was used in this study. Seeds were surface-sterilized with $5 \%$ javel water for $1 \mathrm{~h}$, then germinated on wet filter paper at $30^{\circ} \mathrm{C}$ for $24 \mathrm{~h}$ under dark conditions. Rice seedlings were grown in a green house with a cycle of $16 \mathrm{~h} \mathrm{light}\left(28^{\circ} \mathrm{C}\right)$ and $8 \mathrm{~h}$ dark $\left(23^{\circ} \mathrm{C}\right)$ and a humidity of $30 \%$ for 10 days. Hogland solution was supplied every two days to provide whole nutrition to the seedlings. To investigate the tissue expression pattern of rice 14-3-3 genes, some seedlings were transplanted to the field and grown in normal season. Samples of roots, stems, leaves, glumes before pollination, 2-day glumes after pollination, and 15-day-old seeds were harvested in adult age. For various stress treatments, the roots of 10-day-old rice seedlings were submerged separately in aqueous solutions containing ET (an ethylene-releasing compound, ethephon), ABA (abscisic acid), SA (salicylic acid), IAA (indole-3-acetic acid) or JA (jasmonic acid) for $3 \mathrm{~h}$ and in different concentrations of $\mathrm{H}_{2} \mathrm{O}_{2}, \mathrm{NaCl}, \mathrm{CuSO}_{4}$ or $\mathrm{CdSO}_{4}$ aqueous solutions for $6 \mathrm{~h}$, respectively. Other seedlings were subjected to cold $\left(4^{\circ} \mathrm{C}\right)$ or heat treatment $\left(42^{\circ} \mathrm{C}\right)$ for $6 \mathrm{~h}$. All prepared materials were immediately frozen in liquid nitrogen and stored at $-80^{\circ} \mathrm{C}$ until use.

Database searching and sequence analysis. Protein sequences of OsGF14s were acquired from a query search in the BLAST program at NCBI (http://www.ncbi.nlm.nih.gov/blast/) using the amino acid sequence of the reported OsGF14f. DNA sequences of rice 14-3-3 genes and $1500 \mathrm{bp}$ ahead of their transcriptional initiation sites were collected from the NCBI database. The genome organization and map location were investigated by the corresponding genome sequence with the map viewer at NCBI (http://www. ncbi.nlm.nih.gov/mapview/). The amino acid sequences of OsGF14s were initially aligned using the program ClustalX (ver 1.81) with default gap penalties and further adjusted by GeneDoc program. The unrooted phylogenetic tree was constructed by the neighborjoining method using the same software and displayed with Treeview (ver 1.6.6). Protein structures were viewed using the Swiss PDBview program (http://www.expasy.ch/spdbv) and RasMol software (http://www.rasmol.org). The molecular weights and isoelectric points of OsGF14s were predicted by the Compute $\mathrm{pI} /$ $\mathrm{Mw}$ tool program (http://cn.expasy.org/tools/pi tool.html). The promoters of $O s G F 14 s$ were analyzed by PLACE program (http:// www.dna.affrc.go.jp/PLACE/signalscan.html).

Cloning of the whole ORFs of OsGF14s and semiquantitative RT-PCR assay. Total RNA was extracted from various rice tissues and treated seedlings using the RNeasy Plant Mini Kit according to the instructions provided by the manufacturer. cDNA was synthesized with $1 \mathrm{mg}$ purified total RNA. PCR amplification was carried out using primers designed against the whole ORFs of rice 14-3-3 genes. The amplified fragments were excised from agarose gels, purified, cloned into pMD18-T vector and sequenced. For semiquantitative RT-PCR assays, a DNase I treatment was followed by RNA extraction to remove traces of contaminating DNA. These DNA-free RNA samples were quantified based on their absorbance at $260 \mathrm{~nm}$, then carefully diluted to equal concentrations. The RNA quality was checked by agarose-formaldehyde gel electrophoresis. Reverse transcriptions were performed using $1 \mathrm{mg}$ of purified total RNA. One-twentieth of the cDNA products were used for PCR amplification. A $500 \mathrm{bp}$ fragment of the constitutively expressed Actin gene in rice was used as a control. Seven pairs of specific primers were used in the semiquantitative RT-PCR assay, as follows: $O s G F 14 b, 5$ '-GAGACTTGGCATTTGTAGA-3' and 5'AGTAGCTTAAAGGCGAGA-3'; OsGF14c, 5'-CGCCACCGAA GTAATCCC-3' and 5'-TCGACCGCTACCACCCTA-3'; OsGF14d, 5'-CTCAAGATGAAGGGCGACTA-3' and 5'-GGCATCGTGGAA CAAGAAAA-3'; OsGF 14e, 5'-GAGAAGCCGCAGCCACTGAG AAA-3', and 5'-TGAAACGATAACCCACAGCACAT-3'; OsGF14f, 5'-CTAAGTTTGCGTTGAAGA-3' and 5'-TACAGCAGGTAGCT GAGTA-3'; OsGF14g, 5'-CCCATTCGTCGCCTCCTGTCC-3' and 5'-AGTCCTTCCGCACCCCACCAC-3'; Actin, 5'-TGACGGAGC GTGGTTACTCAT-3' and 5'-GCAATGCCAGGGAACATAGTG-3'. All primers were located in different exons so as to differentiate the amplification of synthesized cDNA from the amplification of genomic DNA. The PCR products were confirmed by sequencing. We also performed experiments to determine the appropriate cycle number so that not only was the amplification product clearly visible on the agarose gel and could be quantified, but also the 
amplification was in the exponential range and did not reach a plateau (data not shown). Therefore, we can guarantee the linearity between the amount of input RNA and the final RT-PCR products. Finally, 26 cycles were performed for the amplification of the cDNA fragments of all OsGF14 and Actin, with the exception of OsGF14g (29 cycles). All RT-PCR expression assays were performed and analyzed at least three times in independent experiments.

Images of the RT-PCR ethidium bromide-stained agarose gels were acquired with the ImageMaster ${ }^{\mathrm{TM}}$ VDS system (Pharmacia Biotech) and quantification of the bands was performed by Quality one software (ver 4.4.0). Band intensities were expressed in relative absorbance units. The ratio between sample RNA and Actin was calculated to normalize for initial variations in the sample concentration.

Functional complement assay. The functional complement assays were performed using van Heusden's strategy by testing the ability of the rice 14-3-3 isoforms to complement the lethal disruption of the two Saccharomyces cerevisiae 14-3-3 genes bmh1 and bmh2 (van Heusden et al., 1996). The yeast strains and plasmids used in this study were kindly provided by Dr. van Heusden. They are: wild type strain GG582-5D (haploid leu2-3,112 ura3-52 trp1-92 his4), mutant strain GG1305 (MATa leu2-3,112 ura3-52 trp1-92 his4 bmh1::LEU2 bmh2::APT1 (YCplac33[BMH1])), pYES-TRP[BMH1] and $\mathrm{pYES}-\mathrm{TRP}$.

Rice 14-3-3 ORFs were obtained from corresponding pMD-

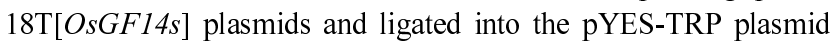
after digestion with $\mathrm{XhoI}$. The galactose-induced promoter of pYES-TRP plasmid could control the expression of foreign 14-3-3 genes. Using the lithium acetate method (Gietz et al., 1995), the wild type strain GG582-5D was transformed with pYES-TRP, while GG1305 was transformed with pYES-TRP, pYES-TRP[BMH1] or various $\mathrm{pYES}-\mathrm{TRP}[O s G F 14 s]$ plasmids. The transformants were screened with SD/Glu/-Trp. Several positive colonies were shaken in the liquid $\mathrm{SD} / \mathrm{Glu} /-\mathrm{Trp}$ medium until $\mathrm{OD}_{600}$ reached $1.00 .5 \mathrm{ml}$ of Serial $1: 10$ dilutions were spotted onto SD/Gal/-Trp medium with 5-fluoroorotic acid (5-FOA) $(1 \mathrm{mg} / \mathrm{ml})$ and SD/Glu/-Trp medium without 5-FOA (as a control). Three independent experiments were performed and similar results were obtained.

\section{Results}

The 14-3-3 gene family in rice. Examining the rice genome revealed eight open reading frames encoding proteins which are highly similar to previously published $14-3-3$ proteins in amino acid sequence. In accordance with previously used nomenclature, we designated them as $O s G F 14 a-h$ respectively. The basal information of all OsGF14 proteins was shown in Table 1 .

The OsGF14 genes encode proteins with calculated $\mathrm{M}_{\mathrm{r}}$ ranging from $25.9 \mathrm{kD}$ to $29.9 \mathrm{kD}$ and estimated $\mathrm{p} I$ ranging from 4.71 to 4.96 , similar to previously reported 14-3-3 proteins. An alignment of deduced OsGF14 proteins is shown in Fig. 1A. The amino acid sequences are highly conserved except in the C-terminal and N-terminal regions. Although the OsGF14h isoform is the most divergent from other members, adding this protein into the alignment did not change the pattern of the conserved block. From homology modeling analyses, we predicted that OsGF14 proteins have a similar three-dimensional conformation to the human zeta isoform, whose threedimensional structure was successfully determined in a previous study (Liu et al., 1995). The human zeta isoform structure features nine antiparallel $\alpha$-helices and is a dimer (Fig. 1C). From our analyses, we observed that all of the $\alpha-$ helical regions are conserved in OsGF14 proteins with the exception of OsGF14h, which seemed to lose part of the sequence in the sixth and seventh $\alpha$-helices. Additionally, conservation among OsGF14 proteins was also supported by the percentage of amino acid and ORF identity, which varied from 46.1 to $95.4 \%$ and 55.8 to $85.3 \%$ respectively.

Plant 14-3-3 proteins have been divided into $\varepsilon$-like groups and non- $\varepsilon$ groups based on phylogenetic clustering in previous studies (Wu et al., 1997; Sehnke et al., 2002). In the model organism Arabidopsis, the non- $\varepsilon$ isoforms include AtGF14 $\chi$, $\omega, \psi, v, \lambda, \nu, \kappa$, while the $\varepsilon$ isoforms include AtGF14 $\mu, \varepsilon, o, 1$, $\pi$ (Wu et al., 1997; Rosenquist et al., 2001). Intriguingly, examining the gene structures of Arabidopsis 14-3-3 genes revealed two completely distinct splicing patterns consistent

Table 1. 14-3-3 proteins in rice

\begin{tabular}{cccc}
\hline Name & $\begin{array}{c}\text { Amino acid } \\
\text { (length) }\end{array}$ & Accession ${ }^{\mathrm{a}}$ & Chromosome location $^{\mathrm{b}}$ \\
\hline OsGF14a & 264 & AAO72553 & $\mathbf{8}(23743186-23745001)$ \\
OsGF14b & 262 & AAB07456 & $\mathbf{4}(23079516-23081945)$ \\
OsGF14c & 256 & AAB07457 & $\mathbf{8}(20805672-20807258)$ \\
OsGF14d & 265 & AAB07458 & $\mathbf{1 1}(19672803-19675261)$ \\
OsGF14e & 262 & CAB77673 & $\mathbf{2}(22354735-22356526)$ \\
OsGF14f & 260 & AAK38429 & $\mathbf{3}(28611035-28613095)$ \\
OsGF14g & 242 & BAD73105 & $\mathbf{1}(5920891-5932738)$ \\
OsGF14h & 230 & ABA94733 & $\mathbf{1 1}(23052640-23048081)$ \\
\hline
\end{tabular}

${ }^{\mathrm{a}}$ GenBank accession number for each protein.

${ }^{\mathrm{b}}$ Location indicates the position of the first and last nucleotide of ORF of each gene in chromosome. 
(A)
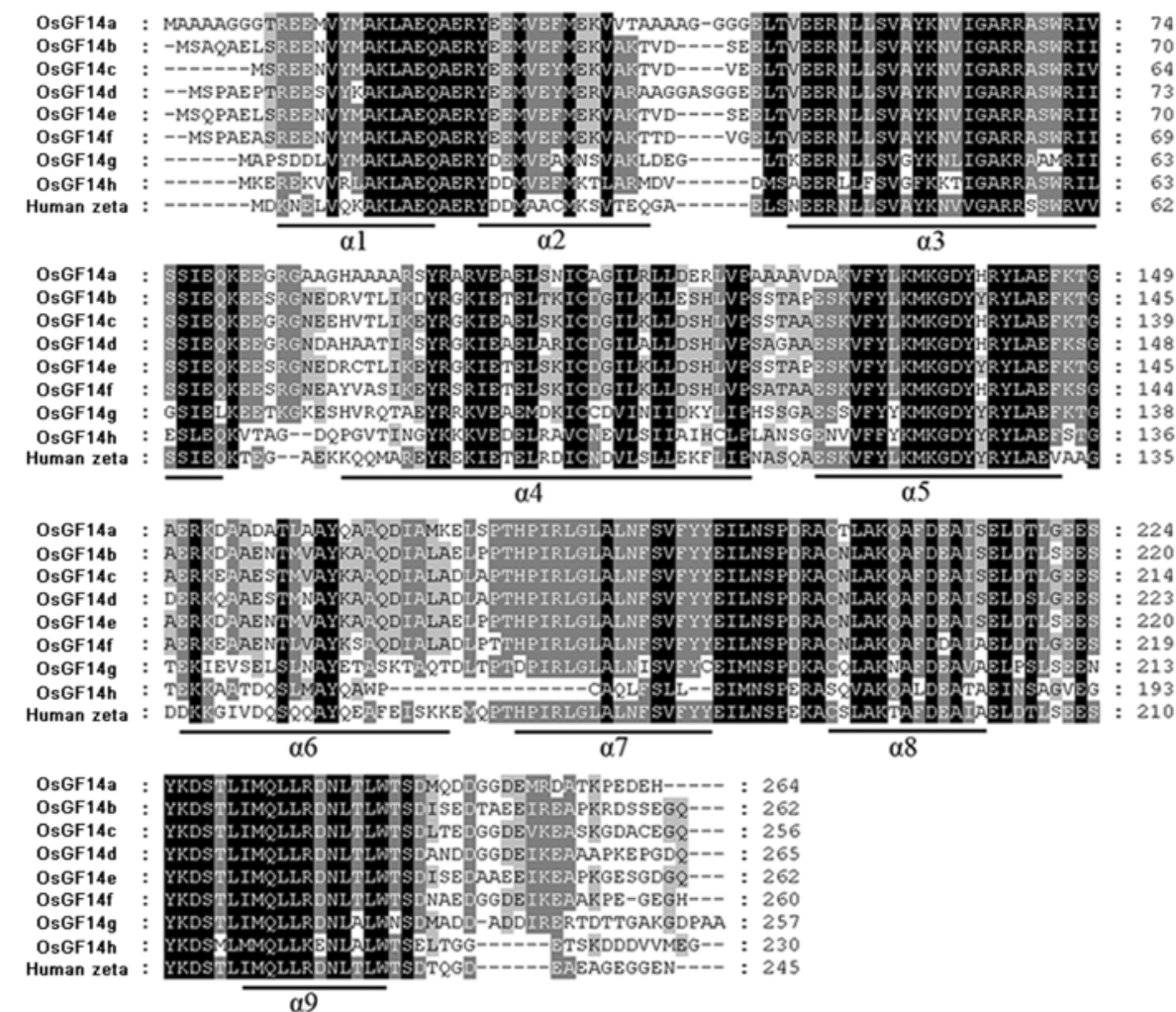

(B)

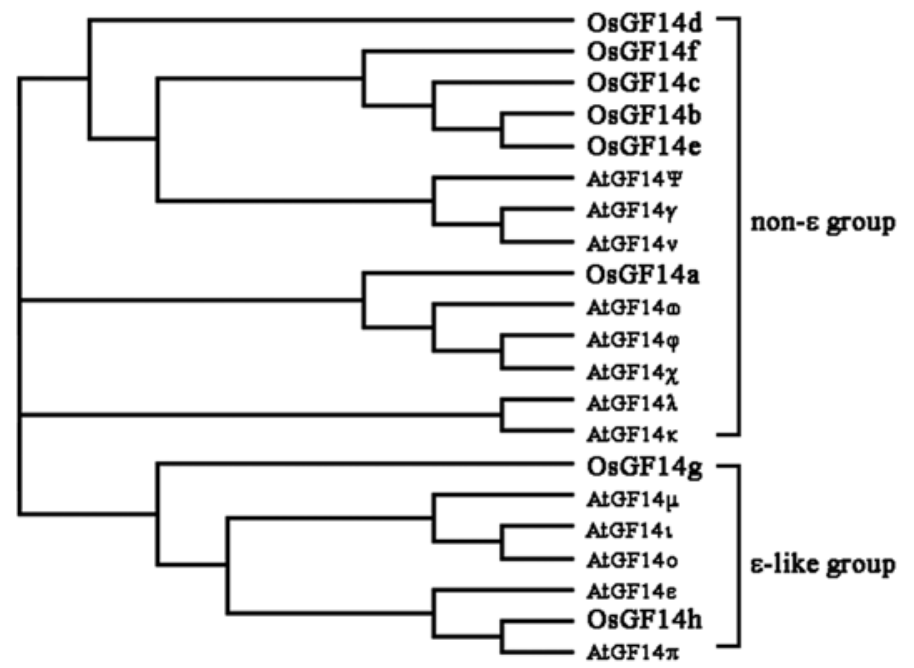

(C)

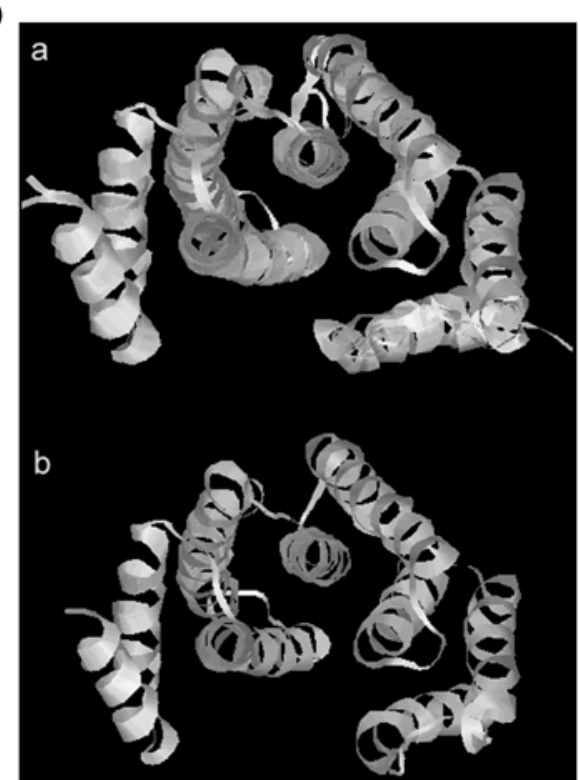

Fig. 1. Sequence alignment of OsGF14 proteins, structure prediction and phylogenetic tree construction. (A) Multiple sequence alignment was performed with the ClustalX program. The amino acid residues identical among the sequences are indicated in black box, while similar residues are shown in grey boxes. Dashes indicate gaps in the sequences to allow for maximal alignment. The regions of the conversed antiparallel $\alpha$-helices are underlined. (B) Unrooted phylogenetic tree was constructed with the ClustalX program using the neighbor-joining method. All sequences analyzed were clustered in two groups. The accession numbers of various

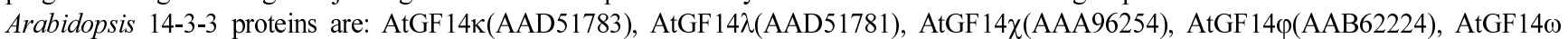
(AAA96253), AtGF14v(AAD51782), AtGF14v(AAB62225), AtGF14 $\psi(A A A 96252)$, AtGF14 $\mu(A A D 51784)$, AtGF141(AAK11271),

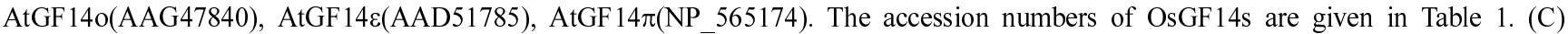
Homology modeling was completed with Swissmodel. The known structure of human 14-3-3 zeta isoform (PDB accession number: P63104) is shown on the top (a) and the predicted OsGF14f is shown on the bottom (b). 


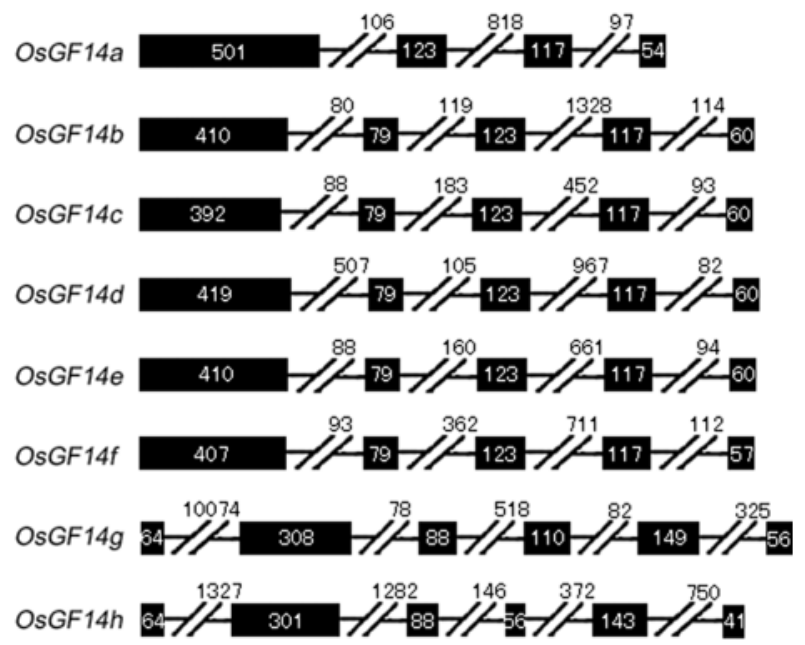

Fig. 2. Structure maps of OsGF14 genes. Exons are shown as black boxes and introns as double slashes. The sizes of exons are indicated with the number of bases within each box, and the length of each box is in scale with the corresponding number of nucleotide residues. The sizes of introns are indicated on top of the double slashes.

with the $\varepsilon$-like and non- $\varepsilon$ clustering (Rosenquist et al., 2001). To determine whether this classification also exists in rice, the members of the OsGF14 family, together with Arabidopsis 14-3-3 isoforms, were introduced into the phylogenetic tree construction. As shown in Fig. 1B, the tree is clearly divided into two distinct groups: OsGF14a-f together with the Arabidopsis non- $\varepsilon$ isoforms formed one branch of the tree, while OsGF14g and OsGF14h together with the Arabidopsis $\varepsilon$-like isoforms formed the other branch. This result indicates that both $\varepsilon$-like and non- $\varepsilon$ 14-3-3 proteins exist in rice. Of these, OsGF14a-f belong to the non- $\varepsilon$ group and OsGF14g and OsGF14h belong to the $\varepsilon$-like group. The investigation of rice 14-3-3 gene structures also supports the phylogenic clustering. As shown in Fig. 2, the gene structures of OsGF14a through $O S G F 14 f$ are highly similar to their Arabidopsis non$\varepsilon$ homologs, whereas OsGF14h and OsGF14g present completely different splicing patterns from other OsGF14s, which are highly similar to the structures of Arabidopsis $\varepsilon$ group genes. Moreover, the closest relative of OsGF14h in the plant kingdom is the typical $\varepsilon$-like Arabidopsis protein GF14epsilon, rather than any of its rice isoforms.

Differential expression of 14-3-3 genes in rice organs. First, we confirmed that six out of eight OsGF14s could be transcribed by reverse transcription and PCR amplification. Although we examined all tissues and seedlings subjected to every treatment mentioned in this study and used several pairs of primers, we still could not find the transcripts of OsGF14a and $O s G F 14 h$. Therefore, the six expressed members were further analyzed. To investigate the expression patterns of the rice 14-3-3 genes in various tissues and at different developmental stages, total RNA from the roots, shoots of 10-
SD YS YR RT ST ML BP AP

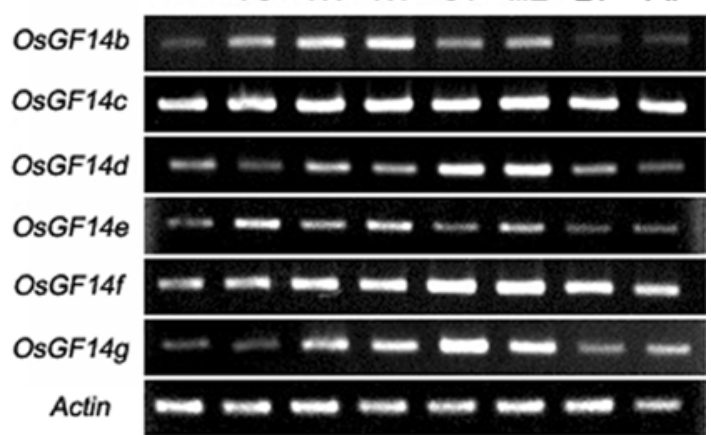

Fig. 3. RT-PCR analysis of OsGF14 transcripts in various tissues. Total RNA isolated from 15-day-old seeds (SD), shoots of 10-day-old seedlings (YS), roots of 10-day-old seedlings (YR), roots of adult rice (RT), stems of adult rice (ST), mature leaves of adult rice (ML), glumes before pollination (BP), 2-day glumes after pollination (AP) were subjected to semiquantitative RT-PCR assay. The PCR products were separated in $1 \%$ agarose gel and stained with ethidium bromide. The constitutively expressed rice Actin gene was chosen as a control and equal amounts of Actin were detected in all lanes.

day-old rice seedlings and many tissues of adult rice (described in Materials and Methods) were subjected to a semiquantitative RT-PCR assay using gene specific primers. As shown in Fig. 3, the $O s G F 14 b$ transcripts were abundant in roots, but low in stems and leaves, and almost absent in seeds and glumes. $O s G F 14 \mathrm{C}$ exhibited a constitutive or housekeeping expression profile, with nearly equal levels in all tissues examined. Transcripts for OsGF14d and OsGF14g showed similar patterns, with the strongest expression in mature leaves and stems and low expression levels in seeds and glumes. The amounts of $O s G F 14$ e transcripts were high in vegetative organs and low in reproductive organs. OsGF14f, the first studied rice 14-3-3 gene, was expressed in all tissues and most strongly in roots, stems, mature leaves and glumes before pollination, while relatively lower levels were observed in developing seeds. All expressed OsGF14 genes gave distinct patterns, indicating that the members of this gene family might undergo different regulation or play different roles during the development process.

Regulation of OsGF14 mRNA levels in response to various stress conditions. Evidence connecting plant 14-3-3 proteins to stress responses came from observed changes in 14-3-3 gene expression under stress conditions. For example, one of the first plant 14-3-3 genes isolated, OsGF14f, was identified as a transcript that accumulated in the callus and seedlings of rice when exposed to high $\mathrm{NaCl}$ concentration or low temperature (Kidou et al., 1993). However, whether all or only specific members in the 14-3-3 family respond to certain environmental stresses has not been studied. Therefore, we examined the transcripts of six expressed $O s G F 14 s$ in response to salinity, temperature, oxidation stresses and toxic level of 
(A)

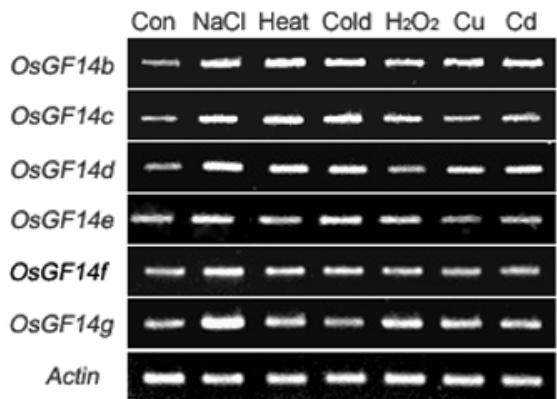

(B)

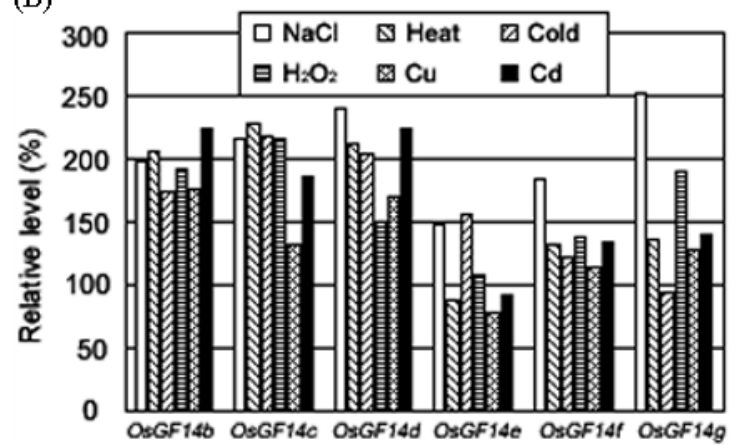

Fig. 4. Expression of $O s G F 14$ genes exposed to various stress treatments. (A) 10-day-old rice seedlings were treated with 200 $\mathrm{mM} \mathrm{NaCl}(\mathrm{NaCl}), 42^{\circ} \mathrm{C}$ heat treatment $(\mathrm{H}), 4^{\circ} \mathrm{C}$ cold treatment (Cold), $10 \mathrm{mM} \mathrm{H}_{2} \mathrm{O}_{2}, 100 \mathrm{mM} \mathrm{CdCl}$ (Cd), $100 \mathrm{mM} \mathrm{CuSO}_{4}(\mathrm{Cu})$ for $6 \mathrm{~h}$; The control (Con) was treated with distilled water. Total RNA were isolated from shoots of the seedlings and subjected to RT-PCR assay. (B) The loading mRNA amounts were standardized by comparison with the PCR product of the rice Actin gene, resulting in relative values of expression intensity.

heavy metals by semiquantitative RT-PCR assay. As shown in Fig. 4, salt stress caused the transcripts of all $O s G F 14 s$ to accumulate. $O s G F 14 g$ exhibited the strongest response and an approximately 2.5 fold increase was observed. Heat treatment resulted in the remarkable enhancement of $O s G F 14 b, O s G F 14 c$ and $O s G F 14 d$ transcripts, with only slight increases in $O s G F 14$, OsGF14f and $O s G F 14 g$ transcripts. When the seedlings were exposed to low temperature $\left(4^{\circ} \mathrm{C}\right)$, the only gene that showed unaffected mRNA levels was OsGF14g. Oxidative stress caused by $\mathrm{H}_{2} \mathrm{O}_{2}$ resulted in the up-regulated transcript levels of OsGF14b, OsGF14c, OsGF14d and OsGF14g. The response of $O s G F 14 s$ to toxic concentrations of the heavy ions $\mathrm{Cu}^{2+}$ and $\mathrm{Cd}^{2+}$ showed similar patterns, with a simultaneous increase in $O s G F 14 b, O s G F 14 c$, OsGF14d and OsGF14g. Generally, some transcription factors play important roles in regulating mRNA levels by binding the promoters of target genes. Analyzing the 5'-flanking sequences of $O s G F 14$, we found the low temperature responsive elements (LTRE) existed in promoter regions of $O s G F 14 b, c$, e, $f$ and copper-response elements (CuRE) located in promoters of $O s G F 14 b, c, d$ and $g$, which were consistent with the RT-PCR results. Besides, other elements related to abiotic stresses, such as drought-
(A)

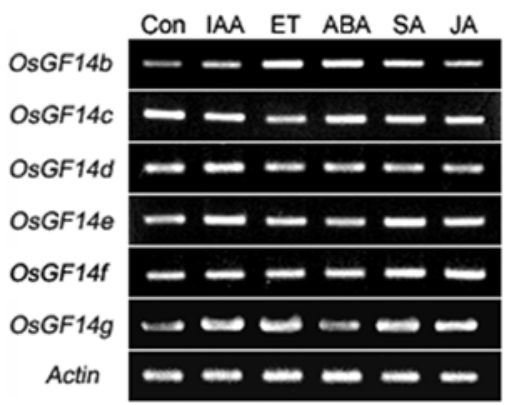

(B)

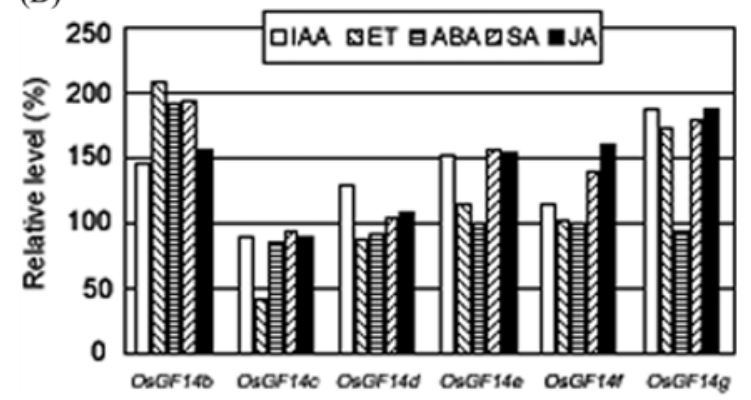

Fig. 5. Effect of different hormones on the expression of OsGF14 genes. (A) 10-day-old rice seedlings were treated with different hormones: $10 \mathrm{mM}$ IAA, $10 \mathrm{mM} \mathrm{GA}_{3}, 2 \mathrm{mM}$ ET, 100 $\mathrm{mM}$ ABA, $5 \mathrm{mM}$ SA, $100 \mathrm{mM}$ JA for $3 \mathrm{~h}$. Total RNA were isolated from shoots of the seedlings and subjected to RT-RCR assay. (B) The loading mRNA amounts were standardized by comparison with the PCR product of the rice Actin gene, resulting in relative values of expression intensity.

responsive elements (DRE) and binding sites of $\mathrm{Myb}$ tramscription factors, also widely distributed during promoter regions of $O s G F 14 s$. In conclusion, our results indicate that the $O s G F 14$ gene family responds to a wide range of stresses and under certain conditions such as salinity stress, cold and heavy iron treatment, some members in the family might be regulated coordinately. At least parts of these responses might be achieved by special transcription activators binding corresponding cis-elements of OsGF14 promoters.

Effects of plant hormones on the expression of OsGF14 genes in rice seedlings. To further study the effects of signaling molecules on expression of the OsGF14 gene family, an RT-PCR assay was also performed using rice seedlings treated with various plant hormones as described in Materials and Methods (Fig. 5). An increase in the mRNA levels of $O s G F 14 b, O s G F 14 d$, OsGF14e and OsGF14g was observed in seedlings treated with IAA compared with control seedlings. In the ET treatment, the transcription of $O s G F 14 b$ and $O s G F 14 g$ were up-regulated, while the $O s G F 14 c$ transcript fell below the control level. ABA treatment specifically increased the expression level of $O s G F 14 b$, whereas JA and SA treatments widely up-regulated the transcription of the $O s G F 14$ gene family, with the exception of $O s G F 14 c$ and 


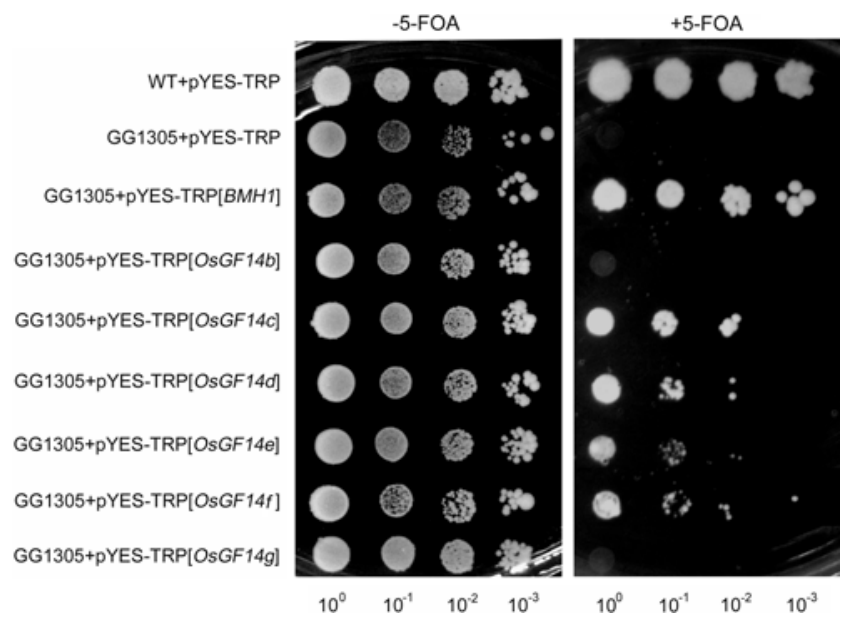

Fig. 6. Complementation effects of OsGF14s on the lethal yeast bmh1 bmh2 double disruption. Yeast strains were transformed with various plasmids. Transformants were shaken in the liquid $\mathrm{SD} /$-Trp medium until equal concentrations were reached. Serial dilutions were spotted onto $\mathrm{SD} / \mathrm{Glu} / \mathrm{-Tr} /-5-\mathrm{FOA}$ medium and $\mathrm{SD} / \mathrm{Gal} /-\mathrm{Trp} /+5$-FOA medium at the same time. The results were acquired by incubating the yeast cells at $30^{\circ} \mathrm{C}$ for 7 days.

OsGF14d. Although not all of mRNA changes could match with corresponding cis-elements predicted by PLACE programe, we did find that the responses to SA and JA have some correlation with the elements in gene 5 '-flanking regions. Our results indicate that each member of the OsGF14 gene family responds in a different manner to plant hormones. Furthermore, these data might provide some clues for further study of the roles of 14-3-3 in plant signal regulation, since most plant hormones are important signaling intermediates.

Functional complementation of the lethal yeast bmhl/ bmh2 double disruption by OsGF14s. In order to investigate whether OsGF14s have the ability to complement the lethal yeast $b m h 1 b m h 2$ double disruption, we employed the yeast complementation system used in van Heusden's study (van Heusden et al., 1996). The yeast strain GG1305 was constructed by disrupting both endogenous $B M H 1$ and $B M H 2$, but it was still kept alive by carrying the plasmid YCplac33 [BMHI] containing an active yeast 14-3-3 gene. When 5-FOA was added into the medium, YCplac33 [BMHI] would be lost and only those yeast cells carrying a functional foreign 14-3-3 could survive. In the present study, GG1305 was transformed with pYES-TRP $[O s G F 14 s]$. All yeast transformants grew well in the SD/Glu/-Trp/-5-FOA medium (Fig. 6). When these transformants were spotted onto SD/Gal/-Trp medium with 5FOA, the loss of YCplac33[BMHI] and the induction of OsGF14 protein expression were initiated. Used as a positive control, it could be clearly observed that pYES-TRP[BMHI] under control of the GAL1 promoter can complement the double disruption. Furthermore, OsGF14c, OsGF14d, OsGF14e and OsGF14f also gave complementation. Among these,
OsGF14c showed the strongest complementation effect. However, OsGF14b and g appeared not to complement under this condition, which might be attributed to poor protein abundance or functional specificity.

\section{Discussion}

The evolution of plant 14-3-3 genes. It is known that 14-3-3 genes constitute the large gene family in plant species. For example, fifteen 14-3-3 homologues were found in the Arabidopsis genome and thirteen of them were found to be expressed. An exhaustive search of the rice genome revealed a 14-3-3 gene family containing eight members. Sequence analysis and a phylogenetic study showed that the 14-3-3 rice isoforms were highly similar to those of Arabidopsis, with a clear classification of $\varepsilon$-like and non- $\varepsilon$ proteins. The discovery of $\varepsilon$-like proteins in rice supported the hypothesis that $\varepsilon$-like proteins exist in monocot species. Therefore, the split into plant $\varepsilon$-like and non- $\varepsilon$ isoforms probably occurred before the divergence of the monocots and dicots. Additionally, the different chromosome locations of most rice GF14 genes suggests that the large gene family might result from regional duplication or chromosome duplication other than tandem duplication.

Rice contains a complex 14-3-3 gene family. As previously reported in Arabidopsis, OsGF14s also constitute a highly conserved protein family in rice. Furthermore, under some stress conditions, several members of this family were regulated coordinately, which indicates their functional similarity. On the other hand, different abilities were presented by OsGF14 proteins in rescuing yeast 14-3-3 double disruption. Although the influence of the different protein expressions in yeast on complementation results can not be excluded, the analyses combining complementation phenotypes with tissue distribution patterns and stress responses might shed some light on the functional diversity of OsGF14s. OsGF14c and OsGF14f, especially OsGF14c, were expressed with high levels in all the tissues examined. Meanwhile, the gene products of OsGF14c and OsGF14f gave strong complementation in the lethal yeast bmh1:bmh2 double disruption. These data indicate that both proteins might have fundamental 14-3-3 functions and are involved in basic physiological processes required for the sustenance of living cells. OsGF14d and OsGF14e could also perform similar functions to yeast 14-3$3 \mathrm{~s}$ in the complementation assays. However, these two genes exhibited different tissue distributions and stress response patterns, suggesting that each of them might possess some special traits apart from the fundamental 14-3-3 functions, or might undergo different regulations during developmental processes or environmental stresses. OsGF14b and OsGF14g gave no complementation in our study. Considering the fact that these two genes were induced under almost all stresses and hormones, and that the sequence of OsGF14g is 
somewhat distinct from other OsGF14s, we suggest that they might perform very specific functions under abnormal conditions or special developmental stages rather than fundamental 14-33 functions. Additionally, it is intriguing that no expression of the OsGF14a and OsGF14h genes was found in our study. OsGF14a was reported as a WIN2-like protein which interacted with a stress-regulated protein kinase and a stressregulated 14-3-3 protein OsGF14f in a yeast two-hybrid assay using a mixed cDNA library generated from various stress treated rice (Cooper et al., 2003a; Cooper et al., 2003b). OsGF14h, belonging to the $\varepsilon$-like group, is the most divergent member of all OsGF14s and lacks approximately 20 amino acids in the 6 and $7 \alpha$-helix regions. Therefore, the absence of the two transcripts in this study might be attributed to their very specific expression profiles, either regarding developmental stages, tissues or stress conditions, although possible expression of these two genes could not be ruled out.

Rice 14-3-3 proteins widely respond to stress conditions and hormones. Previously, OsGF14f was found to accumulate in rice calluses and seedlings under salinity and cold treatment. In the present study, we found that the accumulation introduced by the two stresses exists in almost the whole gene family. Moreover, other stress conditions, including heat, oxidation and heavy metals, could also bring about different effects on OsGF14 gene expression. However, the exact functions of 14-3-3 proteins under these stress conditions remain unknown. The interaction of 14-3-3 proteins with other proteins might provide clues for future study. For instance, it is now clear that $14-3-3$ proteins interact directly with the regulatory $\mathrm{C}$-terminus of plasma membrane $\mathrm{H}^{+}$ATPase, resulting in displacement of the autoinhibitory domain of the enzyme and hence activation (Jahn et al., 1997). The plasma membrane $\mathrm{H}^{+}$-ATPase acts as a primary transporter by pumping $\mathrm{H}^{+}$out of the cell, thus creating a $\mathrm{pH}$ and electrical potential gradient across the plasma membrane. The gradient is then utilized as the driving force for the secondary transport of ions and nutrients into and out of cells (Michelet and Boutry, 1995). When plants encounter stresses, the expression of 14-3-3 genes is up regulated and thus more proteins become available to activate the $\mathrm{H}^{+}$pump, which is crucial for the defensive systems that plants have developed against external adverse influences. Here we provide one example by which 14-3-3 proteins exert their power against stresses. However, as we know, 14-3-3 proteins participate in many physiological processes. Therefore, they are more likely to be utilized simultaneously to resist environmental assaults, albeit in different manners.

Preliminary information for the relationship between OsGF14 members and phytohormones is also provided in this study. Transcripts of $O s G F 14$ genes were regulated in different ways by hormones. It is well known that many responses to biotic and abiotic stresses in plants are mediated by plant hormones. For example, SA is an essential mediator in pathogen defense (Shah, 2003). Our results showed that 4 out of the 6 rice GF14 genes studied were found to be up regulated by SA treatment, which is consistent with a previous study uncovering the functional role for 14-3-3 proteins in hybrid poplar pathogen resistance (Lapointe et al., 2001). Furthermore, all rice GF14 genes were regulated by more than one type of hormone signal, indicating that each member of this family might participate in multiple signaling pathways.

In conclusion, the identification and initial characterization of the eight rice 14-3-3 isoforms provide us an opportunity to understand the fundamental functions and diversity of 14-3-3 proteins within the organism. Major challenges for the future include: (i) examination of the response of OsGF14s to various stresses at the protein level with isoform-specific antibodies; (ii) monitoring the specificity of the interaction between isoforms and target proteins; and (iii) clarification of the different effects of each OsGF14 protein on the signal pathway(s) through observing the phenotype of transgenic plants with sense and antisense genes. Once these questions are addressed, we should gain a greater understanding of the entire 14-3-3 gene family in rice and other organisms.

Acknowledgments We are grateful to Dr. G. Paul H. van Heusden for providing the yeast strains: GG582-5D and GG1305 and plasmids: pYES-TRP[BMHI] and pYES-TRP. We thank members of the Laboratory of Molecular Biology at Tsinghua University for comments and participation in discussion. This work was supported by grants from the State Key Basic Research and Development Plan of China (2006CB101706) and the National Natural Science Foundation of China (30270753, 30170080, 30370804 and 30370847).

\section{References}

Bachmann, M., Huber, J. L., Athwal, G. S., Wu, K., Ferl, R. J. and Huber, S. C. (1996) 14-3-3 proteins associate with the regulatory phosphorylation site of spinach leaf nitrate reductase in an isoform-specific manner and reduce dephosphorylation of Ser-543 by endogenous protein phosphatases. FEBS Lett. 398, 26-30.

Booij, P. P., Roberts, M. R., Vogelzang, S. A., Kraayenhof, R. and De Boer, A. H. (1999) 14-3-3 proteins double the number of outward-rectifying $\mathrm{K}^{+}$channels available for activation in tomato cells. Plant J. 20, 673-683.

Cooper, B., Clarke, J. D., Budworth, P., Kreps, J., Hutchison, D., Park, S., Guimil, S., Dunn, M., Luginbuhl, P., Ellero, C., Goff, S. A. and Glazebrook, J. (2003a) A network of rice genes associated with stress response and seed development. Proc. Natl. Acad. Sci. USA 100, 4945-4950.

Cooper, B., Hutchison, D., Park, S., Guimil, S., Luginbuhl, P., Ellero, C., Goff, S. A. and Glazebrook, J. (2003b) Identification of rice (Oryza sativa) proteins linked to the cyclin-mediated regulation of the cell cycle. Plant Mol. Biol. 53, 273-279.

Dougherty, M. K. and Morrison, D. K. (2004) Unlocking the code of 14-3-3. J. Cell Sci. 117, 1875-1884.

Fu, H., Subramanian, R. R. and Masters, S. C. (2000) 14-3-3 proteins: 
structure, function, and regulation. Annu. Rev. Pharmacol. Toxicol. 40, 617-647.

Gietz, R. D., Schiestl, R. H., Willems, A. R. and Woods, R. A. (1995) Studies on the transformation of intact yeast cells by the LiAc/SS-DNA/PEG procedure. Yeast 11, 355-360.

Holtman, W. L., Roberts, M. R. and Wang, M. (2000) 14-3-3 proteins and a 13-lipoxygenase form associations in a phosphorylationdependent manner. Biochem. Soc. Trans 28, 834-836.

Igarashi, D., Ishida, S., Fukazawa, J. and Takahashi, Y. (2001) 143-3 proteins regulate intracellular localization of the bZIP transcriptional activator RSG. Plant Cell 13, 2483-2497.

Jahn, T., Fuglsang, A. T., Olsson, A., Bruntrup, I. M., Collinge, D. B., Volkmann, D., Sommarin, M., Palmgren, M. G. and Larsson, C. (1997) The 14-3-3 protein interacts directly with the C-terminal region of the plant plasma membrane $\mathrm{H}(+)-$ ATPase. Plant Cell 9, 1805-1814.

Kidou, S., Umeda, M., Kato, A. and Uchimiya, H. (1993) Isolation and characterization of a rice cDNA similar to the bovine brain-specific 14-3-3 protein gene. Plant Mol. Biol. 21, 191-194.

Lapointe, G., Luckevich, M. D., Cloutier, M. and Seguin, A. (2001) 14-3-3 gene family in hybrid poplar and its involvement in tree defence against pathogens. J. Exp. Bot. 52, 1331-1338.

Liu, D., Bienkowska, J., Petosa, C., Collier, R. J., Fu, H. and Liddington, R. (1995) Crystal structure of the zeta isoform of the 14-3-3 protein. Nature 376, 191-194.

Lu, G, Paul, A. L., McCarty, D. R. and Ferl, R. J. (1996) Transcription factor veracity: is GBF3 responsible for ABA-regulated expression of Arabidopsis Adh? Plant Cell 8, 847-857.

Michelet, B. and Boutry, M. (1995) The plasma membrane $\mathrm{H}^{+}-$ ATPase (A highly regulated enzyme with multiple physiological functions). Plant Physiol. 108, 1-6.

Muslin, A. J. and Xing, H. (2000) 14-3-3 proteins: regulation of subcellular localization by molecular interference. Cell Signal. 12, 703-709.

Qi, W., Liu, X., Qiao, D. and Martinez, J. D. (2005) Isoformspecific expression of 14-3-3 proteins in human lung cancer tissues. Int. J. Cancer 113, 359-363.
Rosenquist, M., Alsterfjord, M., Larsson, C. and Sommarin, M. (2001) Data mining the Arabidopsis genome reveals fifteen 143-3 genes. Expression is demonstrated for two out of five novel genes. Plant Physiol. 127, 142-149.

Rosenquist, M., Sehnke, P., Ferl, R. J., Sommarin, M. and Larsson, C. (2000) Evolution of the 14-3-3 protein family: does the large number of isoforms in multicellular organisms reflect functional specificity? J. Mol. Evol. 51, 446-458.

Schultz, T. F., Medina, J., Hill, A. and Quatrano, R. S. (1998) 143-3 proteins are part of an abscisic acid-VIVIPAROUS1 (VP1) response complex in the Em promoter and interact with VP1 and EmBP1. Plant Cell 10, 837-847.

Sehnke, P. C., Rosenquist, M., Alsterfjord, M., DeLille, J., Sommarin, M., Larsson, C. and Ferl, R. J. (2002) Evolution and isoform specificity of plant 14-3-3 proteins. Plant Mol. Biol. 50, 1011-1018.

Shah, J. (2003) The salicylic acid loop in plant defense. Curr. Opin. Plant Biol. 6, 365-371.

Toroser, D., Athwal, G. S. and Huber, S. C. (1998) Site-specific regulatory interaction between spinach leaf sucrose-phosphate synthase and 14-3-3 proteins. FEBS Lett 435, 110-114.

van Heusden, G. P., Griffiths, D. J., Ford, J. C., Chin, A. W. T. F., Schrader, P. A., Carr, A. M. and Steensma, H. Y. (1995) The 14-3-3 proteins encoded by the BMH1 and BMH2 genes are essential in the yeast Saccharomyces cerevisiae and can be replaced by a plant homologue. Eur. J. Biochem. 229, 45-53.

van Heusden, G. P., van der Zanden, A. L., Ferl, R. J. and Steensma, H. Y. (1996) Four Arabidopsis thaliana 14-3-3 protein isoforms can complement the lethal yeast bmh1 bmh2 double disruption. FEBS Lett. 391, 252-256.

Wang, W. and Shakes, D. C. (1996) Molecular evolution of the 14-3-3 protein family. J. Mol. Evol. 43, 384-398.

Wu, K., Rooney, M. F. and Ferl, R. J. (1997) The Arabidopsis 143-3 multigene family. Plant Physiol. 114, 1421-1431.

Zhang, H., Wang, J., Nickel, U., Allen, R. D. and Goodman, H. M. (1997) Cloning and expression of an Arabidopsis gene encoding a putative peroxisomal ascorbate peroxidase. Plant Mol. Biol. 34, 967-971. 